\title{
An immunoregulatory molecular complex with five active sites
}

\section{from N.A. Mitchison}

THE cellular immunology group at Yale University has just published a very important claim'. It comes in several parts, of which the most important is to have discovered a new suppressive transmitter or 'factor' with five distinct active sites.

Antibody production in the immune response is believed to be regulated in part by a cascade of interacting $T$ cells which produce soluble transmitters that act on others in the chain and ultimately suppress the activity of the helper $T$ cells that induce the proliferation of the antibodyproducing $\mathrm{B}$ cells. The new transmitter is made by two cells at the end of the suppressor $\mathrm{T}$ cell cascade, each of which releases a single peptide chain (or, just possibly, a very stable assembly of chains). The two chains assemble to make up a complex which acts in a suppressive manner on the target of suppression, a helper $T$ cell. Two of the five active sites on this two-chain molecule are necessary for assembly of the chains. One of these sites is a product of the $\mathrm{H}-2 \mathrm{~J}$ locus of the major histocompatibility complex, made by a cell which bears the same product on its surface. $\mathrm{H}-2 \mathrm{~J}$ is well known as a marker on suppressor $\mathrm{T}$ cells and their products, so it is no surprise to find it turning up here. The other site is on the other chain made by an $\mathrm{H}-2 \mathrm{~J}^{-}$cell, and it binds the $\mathrm{H}-2 \mathrm{~J}$ product on the $\mathbf{H}-2 \mathbf{J}^{+}$chain.

The $\mathrm{H}-2 \mathrm{~J}^{+}$chain also has an antiidiotype site, which binds to an idiotype on the surface of the target cell. Idiotypes are antigenic determinants of the antigenbinding sites of antibodies. They can also be detected on $T$ cells where they are believed to be associated with the antigenbinding site of the T cell receptor. Because of their association with the antigenbinding site, they are thought to be the target of antigen-specific regulation of the immune response. However, it is a little surprising to encounter them at the end of the suppressor cascade, since they have more usually been associated with its induction rather than with its final action. (Gershon et al. do not use the term idiotype; I suspect this is because they believe that products similar but not identical to immunoglobulin are involved, which are encoded by a family of related genes on the same chromosome and immediately to the right of the immunoglobulin genes. Whether or not they are correct in this belief, the idiotypic nomenclature seems appropriate and is in general use for markers identified by the mouse strains they use.)

The other chain, in addition to the $\mathrm{H}-2 \mathrm{~J}$ -

N.A. Mitchison is in the Department of Zoology, University College London, London WCIE 6BT. binding site, carries an antigen-binding site and an effector site that mediates the suppression of the target cell. The antigenbinding site presumably makes a bridge via antigen to the target cell, and thus helps the transmitter find its appropriate target which it then suppresses, Gershon et al. suggest, by cleaving to generate a toxic peptide. They have in mind the way diphtheria toxin acts, and more immediately a cleavage mechanism to yield a toxic product which has recently been demonstrated for the products of a T suppressor cell clone ${ }^{2}$.

It is further proposed that this transmitter is only one among a class of similar molecular assemblies, all of which are composed of a pair of chains, one with an anti-idiotype binding site and the other with effector activity. In another publication ${ }^{3}$, the Yale group describes another such factor which acts earlier in the suppressor cascade. The function of this one is to induce activity in the terminal cells of the cascade, and accordingly its effector site has an activating rather than a suppressive effect. Thus, as a general rule, the anti-idiotype chain can be regarded as a 'schlepper' or carrier, targetting the effector activity to the $T$ cell bearing the appropriate antigenic specificity.

The importance and originality of this part of the claim is as follows. In the first place, it provides fresh insight into the linkage between cells within the immunoregulatory circuits and cascades. If transmitters really work through generating active peptides in the way that Cantor, Gershon and their colleagues propose, then a whole new field of cell biology opens up concerning the mode of action of these peptides. It will be reminiscent of the study of bacterial toxins and colicines, but different insofar as it will deal with physiological interactions rather than attack and defence. Furthermore, the idiotype- and antigen-binding activity of these transmitters makes sense only if they act at a fair distance. It is refreshing to find this kind of evidence at a time when the case for interleukin-2, hitherto the strongest candidate for a long-distance transmitter between $\mathrm{T}$ cells, is weakening 4.5 . What is more generally agreed is that it profits little to debate whether these transmitters operate physiologically or are in vitro artefacts. After all, the neurophysiologists have been debating the same question over substance $\mathrm{P}$ for the last fifty years, and the molecular biologists seem to be on the point of building enough of these immunologically active transmitters to answer some really decisive questions. This, in fact, is another reason why the present claim is so important: the new idiotype- and $\mathrm{H}-2 \mathrm{~J}-$ binding sites should provide useful handles for grabbing these polypeptides in transcription experiments.

But surely what is most important is to have brought together the idiotope- and antigen-recognizing systems. Hitherto authorities such as Sercarz and Janeway (himself a member of the Yale group) have argued that these two activities are mediated by different $T$ cells ('Ts1 and Ts2'), acting independently of one another and converging only at the end of the regulatory cascade. What is now proposed is that within the suppressor cascade at least, the two activities are not separate.

This brings us to the second part of the claim, advanced in the present publication with further details promised later, that the anti-idiotype repertoire of $T$ cells is acquired in the thymus. Thus the presence of an $(A \times B) F_{1}$ thymus graft in an A-strain mouse enables its $T$ cells to produce a factor which recognizes immunoglobulinlinked products on B-strain cells under circumstances where normal A-strain T cells would not be able to do so. This again runs contrary to current doctrine, that the $T$ cell receptor repertoire for recognition of idiotypes is largely independent of the thymus.

How convincing are these claims? The idea that a single factor may carry both $\mathrm{J}$ and an antigen-binding site was implicit in the very first work on $\mathbf{J}$, and has been extended recently to include a factor with $\mathrm{J}$-binding and antigen-binding activities ${ }^{6}$. Incidentally, if Zembala et al. are correct, the target of the $\mathrm{J}$ - and antigen-binding factor is itself still a suppressor cell, not a helper. What is new is that five activities can all go together, including two from distinct chromosomes $(\mathrm{H}-2 \mathrm{~J}$ is on chromosome 19 , immunoglobulin on chromosome 12) that appear to be on the same chain. The Yale group indeed recognizes this difficulty, and has no solution to offer at present. They cite other work on two-chain suppressor factors, but the analogy is surely misleading since these involve chains separately encoded by chromosomes 12 and 19.

Perhaps the main point is this. There is a further part of this study which is still owing. In the publications which I have so far seen, there is no direct stoichiometry of the proposed molecular assembly. Obviously the two chains need to be characterized separately, and their binding to one another and to appropriate substrates examined, either in solution or on appropriate absorbents. It looks as though some of the data could be acquired rather easily, particularly as significant readings can be made from as little as "three individual calculations from each culture condition". Perhaps the Yale group already has these data. Until they are available, the group's claims should be treated with some caution.

\footnotetext{
Filood Yamauchi \& Girshon $J$ exp. Med (in the prese).

Fresno el al. J. exp. Med. 153, 1260 (1981).

Yamauchi et al. J. exp. Med. (in the press).

Kcenc \& Forman J. exp. Med. 155. 768 (1982)

Crispe el al. Transplantn Proc. (in the press).

Zermbala, Asherson \& Golizzi Nature 297, 41 ) (1982).
} 